\title{
Pencil-Beam Fluence Evaluation Based on Monte Carlo Simulations Algorithm of High Energetic Treatment Photons
}

\begin{abstract}
Background: The accurate calculation of doses during external radiotherapy sessions is necessary. Recently, the models used for this purpose have been the point kernel, pencil-beam, and collapsed cone superposition/convolution combination models. Methods: In this study, it is aimed to determine point/pencil-beam kernels to be used in dose calculations. For this purpose, tallying pencil-beam fluence based on Monte Carlo (MC) simulations is investigated by scoring a volume of interest centered in a cubic water phantom at a depth of $10 \mathrm{~cm}$ and the irradiated field of $10 \mathrm{~cm} \times 10 \mathrm{~cm}$. The fluence is calculated for each mono-energetic photon ranging from $0.25-6 \mathrm{MV}$ at increments of $250 \mathrm{keV}$. Results: The output of the four fluence kernels along the depth $\mathrm{Z}$ around the central axis is categorized for both the primary and secondary photons and electrons. Here, a database for pencil-beam kernels is established for each category. For validation purposes, other MC simulations are carried out for fluence calculations as produced by the predetermined poly-energetic spectra for a Varian $6 \mathrm{MV}$ linear accelerator. Conclusions: The net results show a good fit of the two convoluted fluence spectra quantities for both mono-energetic and poly-energetics-based simulations except little singular peaks.
\end{abstract}

Keywords: Electron fluence, Monte Carlo simulation, pencil-beam kernel, photon fluence, solid-state dosimeters

\section{Introduction}

Nowadays, there is a growing awareness with respect cancer patient's dose accuracy in modern dose calculations or dose engines based on various algorithms for radiotherapy applications..$^{[1-4]}$ More accurate equipment is now available for estimating absorbed doses during radiotherapy sessions..$^{[5-8]}$ However, it is still suffered inaccuracies despite the availability of advanced treatment methods. For this purpose, in-patients dose engine is based on predetermined energy fluence on or into the patient. ${ }^{[9]}$ Most of these algorithms are constructed to facilitate dose calculations and mapping in different environments. ${ }^{[1,3]}$ Two of these algorithms are point beam kernel (PK) and pencil-beam kernels (PBK) that have been extensively used. PBK methods superposed the predetermined dose distributions from narrow "pencils" of radiation in water. ${ }^{[4]}$ Some of treatments planning systems are based on PK and PBK for dose deposition calculations..$^{[10-12]}$ One of the examples of using PBK algorithm is presented $\mathrm{in}^{[13]}$ through deconvolution process from experimental data, especially in flattening

This is an open access journal, and articles are distributed under the terms of the Creative Commons Attribution-NonCommercialShareAlike 4.0 License, which allows others to remix, tweak, and build upon the work non-commercially, as long as appropriate credit is given and the new creations are licensed under the identical terms.

For reprints contact: reprints@medknow.com filter-free (FFF) linear accelerator (LINAC) for 3D dose calculations. Uncertainties found in the results depend on particular detail for both calculation of PBK and MC. As an example, implementation of PBK method shows low statistical uncertainty in radiotherapy, ${ }^{[1,3,4]}$ however, it is high for calculation of actual proton doses makes it unsuitable for reflection of dose in some cases. ${ }^{[14]}$ The most interesting approach to PBK fluence has been proposed by ${ }^{[15]}$ which calculated the local spectra via dividing it into high and low energies using small and large cavity theories approximations, respectively. ${ }^{[16]}$

PBK fluence is divided into four groups: ${ }^{[15]}$ primary photons, secondary photons, secondary electrons, and primary electrons. The primary photons would be the photons which remain at a depth $z$ beyond its attenuation. Primary electrons arise from primary photons interacting with LINAC head or phantom. The scattered photons come from primary photon interactions or other scattered particles which contained bremsstrahlung and annihilation photons. At last, electrons which are released from interactions of those scattered photons are converted into scattered electrons. MC How to cite this article: Donya H. Pencil-beam
fluence evaluation based on Monte Carlo simulations
algorithm of high energetic treatment photons. J Med
Sign Sens 2018;8:81-6.
Address for correspondence: Dr. Hossam Donya, Department of Physics, Faculty of Science, King Abdulaziz University, Jeddah 21589,

Saudi Arabia.

Department of Physics, Faculty of Science, Menoufia University, Shibin El-Koom, Egypt.

E-mail: hdunia@kau.edu.sa

Website: www.jmss.mui.ac.ir DOI: 10.4103/jmss.JMSS_48_17 
codes are extensively used in radiotherapy due to its high accuracy of dose calculation and dose distribution. ${ }^{[17-19]}$ Excellent results are recently reported between different MC codes for obtaining a good agreement between percent depth dose curves and depth doses. ${ }^{[20]}$

PENELOPE is a code system that performs photon-electron coupled transport process in materials with a wide-ranging energy. ${ }^{[21-24]}$ It is extensively used in the simulation of $x$ rays spectra, gamma rays and electron emission. ${ }^{[24-26]}$ Moreover, it is implemented in complicated geometries for dose application. ${ }^{[27]}$ Recently, updated versions based on PENELOPE package is used like PENEPMA for electron probe microanalysis measurements. ${ }^{[28]}$ In a recent paper, ${ }^{[29]}$ PENELOPE presented excellent improvements for security purposes in coded aperture and pinhole imaging systems.

A large number of impinging particles is required to get sufficient low uncertainty; however, it is time-consuming. For this purpose, high-performance computers or supercomputers nowadays solve this limitation in a more sophisticated computation. ${ }^{[30]}$ Furthermore, non-analog simulations like that using variance-reduction technique (particle splitting and Russian-roulette techniques) are extensively needed for both efficiency and lesser time for calculations. ${ }^{[15,31]}$

Other advantage of the pencil-beam approach is that it could easily evaluate the deviation induced as a result of compensator or wedge. Accordingly, changing pencil-beam kernel would be easily to treat the variations resulted from wedge filtration or off-axis. So that, tuning the incident fluence over time could be extremely useful toward modeling the complex treatments like as intensity modulated radiation therapy (IMRT). ${ }^{[32]}$ Some methods are well-known for creating pencil beam from measurements such as differentiation of both radial relative dose along the central axis..$^{[33,34]} \mathrm{MC}$ is also used for PBK determinations in any materials. ${ }^{[16,35]}$

Our study aimed to implement the analytical methods of dose engine such as PK and PBK algorithms for fluence spectra calculation. Therefore, MC simulations are carried out for two different cases (mono-energetic and poly-energetic spectra calculations). The essence of PBK is to increase the calculation speed and convolve fluence over the field precedent identified.

\section{Materials and Methods}

To obtain the spectrum of the fluence to the point of interest (x, y, z) with a given irradiation field, we can apply a lateral and a convolution integration on energy range $\left[0, E_{\max }\right]$ convolving over the field aperture, as follows: ${ }^{[32]}$

$$
\begin{aligned}
& \Phi_{E}(x, y, z)=\int_{0}^{E_{\max }} \iint \Psi_{E_{\mathrm{D}}}\left(x^{\prime}, y^{\prime}\right) \\
& \phi_{E}^{\mathrm{p}}\left(E_{\mathrm{D}}, x-x^{\prime}, y-y^{\prime}, z\right) \mathrm{d} x^{\prime} \mathrm{d} y^{\prime} \mathrm{d} E_{\mathrm{D}}
\end{aligned}
$$

Where $\Psi_{E_{\mathrm{D}}}\left(x^{\prime}, y^{\prime}\right)$ is the incident energy fluence spectrum with the maximum energy $E_{\max }$. Both of electron fluence kernel $\phi_{E}^{\mathrm{p}}\left(E_{\mathrm{D}}, x-x^{\prime}, y-y^{\prime}, z\right)$ and photon fluence kernel spectrum $\Psi_{E_{\mathrm{D}}}\left(E_{\mathrm{D}}, x-x^{\prime}, y-y^{\prime}, z\right)$ are defined at the position of $(x, y, z)$ for mono-energetic beam of energy $E_{\mathrm{D}}$.

A commercial application of the PBK is the AAA (analytical anisotropic algorithm). ${ }^{[16,33,36,37]}$ The literature on PBKs shows a variety of approaches. ${ }^{[38-44]}$

PBK is scored in cylindrical voxels with a mono-directional pencil-beam impinging on a homogeneous unlimited phantom. Fluence is differentiated using cylindrical coordinates (radius $r$ and depth $Z$ from the entrance point of $P(x, y, z))$ at which is where the pencil beam enters the phantom.

\section{Monte Carlo simulations setup}

\section{Pencil-beam approach}

The geometry of the simulation is depicted in Figure 1, which is saved in a.geo file and shows two different colors, violet and red, representing the air cavity and the water, respectively. Eighty detectors are implemented to simulate the effects of a mono-direction beam for a maximum depth of $40 \mathrm{~cm}$ under the water. Each detector is described by its cylindrical voxel centered on the z-axis of a volume of $26.8 \mathrm{~cm}^{3}$. The source of the photons is set at $1 \mathrm{~m}$ from the air-water surface, emitting mono-directional photons perpendicular to the surface. The voxels in Figure 1 are the volume of interest in the middle cylinder of $0.5 \mathrm{~cm}$. High computing performance (Aziz) technology that is available at King Abdulaziz University, Jeddah, is used for the simulation of the fluence scoring cylindrical voxel regions. The variation of the energy for the mono-energetic beam kernels is from $0.25-6 \mathrm{MeV}$ with an increment of $250 \mathrm{keV}$.

$\mathrm{EABS}_{1}, \mathrm{EABS}_{2}$ and $\mathrm{EABS}{ }_{3}$, cutoff energies for electrons, photons, and positrons, respectively, are set at $30 \mathrm{keV} . W_{\mathrm{cc}}$ and $W_{\mathrm{CR}}$, minimum energy that transfer in bremsstrahlung emission and elastic collisions, respectively, are set at $30 \mathrm{keV}$. The parameters $C_{1}$ and $\mathrm{C}_{2}$ are the average angular deflection between hard elastic events and maximum average fractional energy loss, respectively in a single

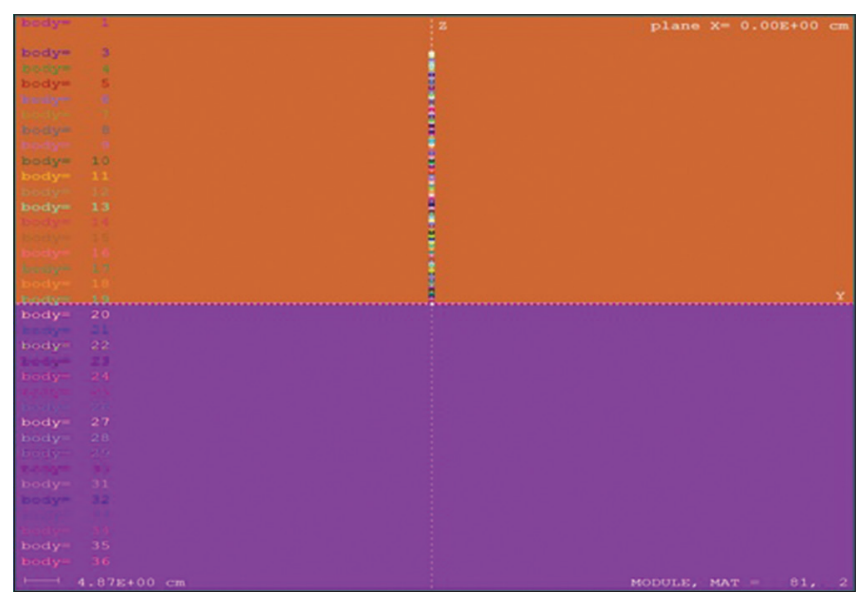

Figure 1: Two-dimensional geometrical view of the simulation 
step. Both values of $C_{1}$ and $\mathrm{C}_{2}$ are set to be small enough at 0.02 for high-resolution particle transport resolution without creating a detailed simulation. ${ }^{[12]} \mathrm{DS}_{\mathrm{MAX}}$ is the maximum step length for electrons which should equal to one-tenth of voxel size, ${ }^{[12]}$ is set at $0.05 \mathrm{~cm}$. The number of simulated primary photons, NSIMSH, is set at $20 \times 10^{6}$ particles.

\section{Results and Discussion}

The fluence kernels of the different categories are represented for both cylindrical radius $r(\mathrm{~cm})$ and the depth $Z(\mathrm{~cm})$ in Figure 2a-d. The incident energy is set at $6 \mathrm{MeV}$. As shown in Figure 2a, the primary photon kernel is only one voxel wide. It is clear that primary photon kernel contains only those photons that have not yet been scattered.

In addition, note that the primary electrons [Figure 2b] are also somewhat limited in a finite volume while the scattered photons and electrons [Figure 2c and d] have a wide span. In the meantime, a problem in the scoring code has rendered this solution almost impossible. As seen in geometry shown in Figure 1, the most voxels defined would not represent the correct value of the incident fluence. For decreasing the uncertainty errors, a source file (penelope.f) in the PENELOPE package is modified to change the rules of scoring according to the interaction history. An alternative compensation is found when the enclosure of the overall system changes. The enclosure usually represents as a giant sphere with a default radius of $3 \times 10^{7} \mathrm{~cm}$. It contains all the defined geometric structures and outer sides of the enclosure would be absolute vacuum such that any tracking history of the particles would stop if they reached the interface of the enclosure. When the change in the enclosure radius is implemented to $3 \times 10^{30} \mathrm{~cm}$, the uncertainty errors of scoring becomes much less visible.

Calculation of the spectra from the pencil-beam kernels

Once PBK database has been established, the net spectrum at any point of an irradiated water slab could be determined through the superposition of the fluence pencil kernels over the field with a known incident fluence. The fluence kernel resulted from the simulation of the polar coordinate system $(r, z)$ is transformed from to the $(\mathrm{x}, \mathrm{y}, \mathrm{z})$ three dimensional space axes. Therefore, energy fluence spectrum could be calculated using convolution in Eq. 1.

\section{A poly-energetic pencil kernel}

A poly-energetic pencil kernel is calculated by taking a beam spectrum for the $6 \mathrm{MV}$ Varian LINAC of reference. ${ }^{[45]}$ The spectrum has been used to simulate the actual spectra of the accelerators. This spectrum is simulated using MC codes (BEAMnrc) and refers specifically to the spectrum of a $6 \mathrm{MV}$ beam on Varian CD 2100 machine. The spectrum is validated by fitting it to that of the actual machines by comparing the measured and simulated depth doses for several field sizes.

Another set of data for a square parallel beam field of $10 \mathrm{~cm} \times 10 \mathrm{~cm}$ has been generated to validate the results of the kernel superposition. Since the size of this simulation is enormous, the parameters are modified, and the simulation is divided into batches to fasten the overall simulation. Geometry of the simulation and the modified simulation parameters $^{[46]}$ are summarized in Table 1.

The geometry of the simulation in a field size of $10 \mathrm{~cm} \times 10 \mathrm{~cm}$ is shown in Figure 3. The volumes of

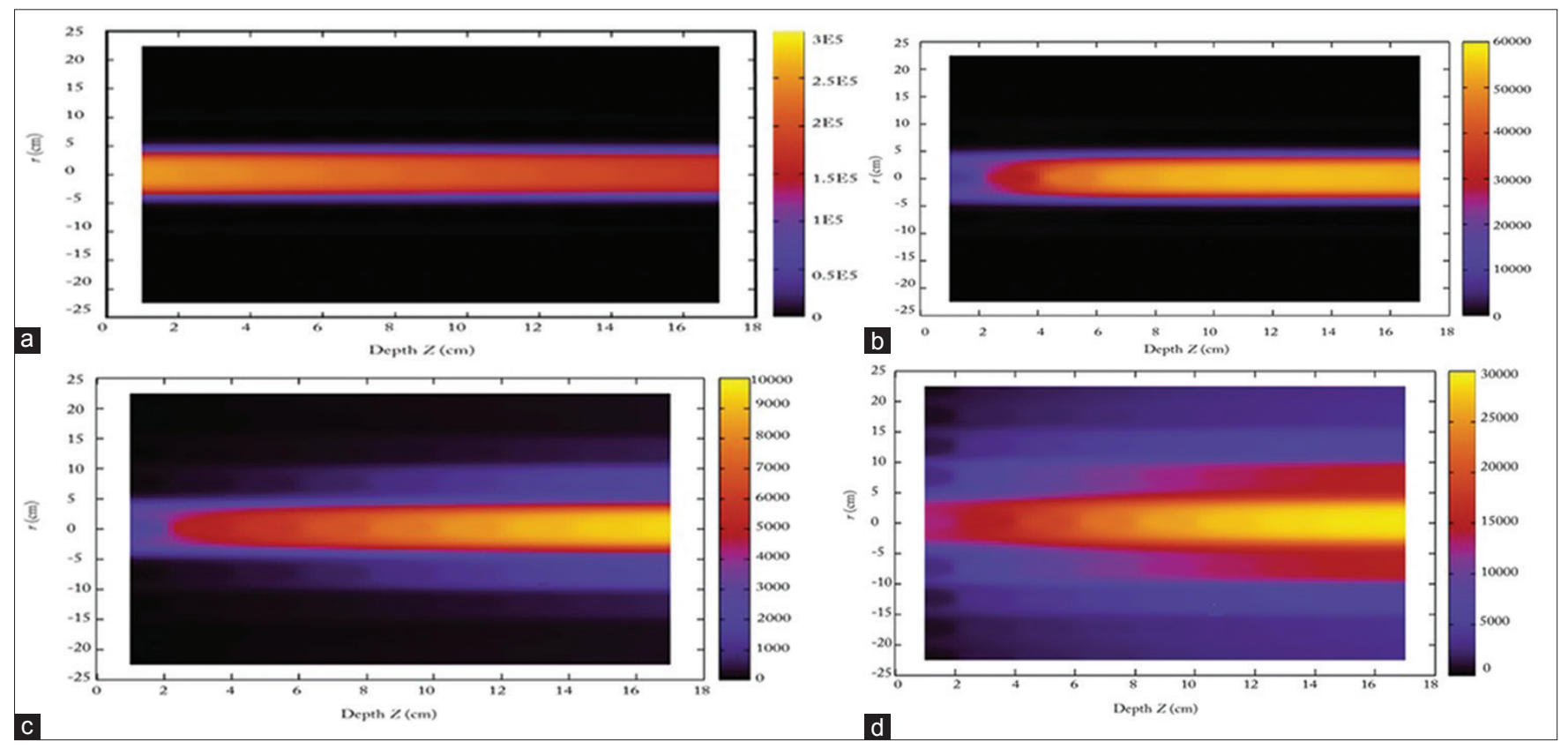

Figure 2: (a) Fluence kernels of the primary photons of mono-directional mono-energetic fluence in water phantom with an incident energy of 6 MV. (b) Fluence kernels of the primary electrons of mono-directional mono-energetic fluence in water phantom with an incident energy of 6 MV. (c) Fluence kernels of the secondary photon of mono-directional mono-energetic fluence in water phantom with an incident energy of 6 MV. (d) Fluence kernels of the secondary electrons of mono-directional mono-energetic fluence in water phantom with an incident energy of $6 \mathrm{MV}$ 


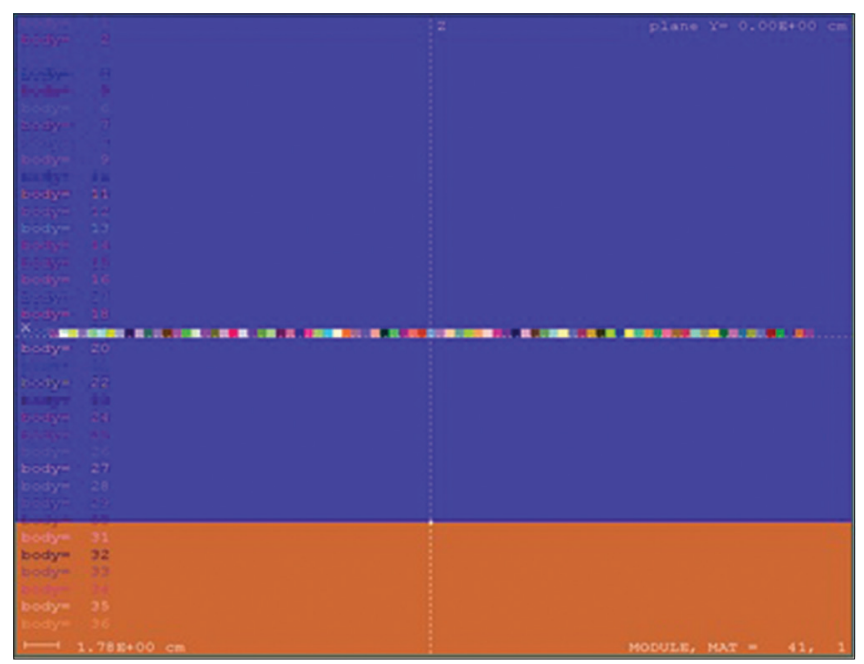

Figure 3: Simulation geometry of $10 \mathrm{~cm} \times 10 \mathrm{~cm}$ field size, volumes of interest are put in a depth of $10 \mathrm{~cm}$ perpendicularly to the axis

Table 1: Simulation parameters used for fluence calculation based on poly-energetic pencil beam kernel

\begin{tabular}{lc}
\hline Parameter & Kernel values \\
\hline EABS $_{1}$ & $80 \mathrm{keV}$ \\
EABS $_{2}$ & $30 \mathrm{keV}$ \\
EABS $_{3}$ & $80 \mathrm{keV}$ \\
$W_{\mathrm{CR}}$ & $30 \mathrm{keV}$ \\
$W_{\mathrm{CC}}$ & $30 \mathrm{keV}$ \\
$C_{1}$ & $5 \%$ \\
$C_{2}$ & $5 \%$ \\
DSMAX & $0.1 \mathrm{~cm}$ \\
NSIMSH & $2 \times 10^{9}$ particles \\
\hline
\end{tabular}

interest are put at a depth of $10 \mathrm{~cm}$ perpendicular to the axis. Thus, a profile of the fluence varying with the position of the volume is presented. Herein, the form of the voxel is defined as a volume of the cube equal to $0.125 \mathrm{~cm}^{3}$.

The profile of the simulated fluence is shown in Figure 4. As shown in Figure 4, the photon fluence is much wider than the fluence of the electrons and positrons.

The positrons lie in almost the region around central axe of incident beam of $5 \mathrm{~cm}$ radius, approximately of the irradiated field as their energy is relatively low. The calculated uncertainty of the fluence is relatively higher at the off-axis region as fewer photons have arrived in these areas. The number of particles simulated in this test should be larger to avoid such high uncertainties.

A comparison of the spectra in the same positions from the simulations and convolutions is made [Figure 5]. The voxel chosen for comparison is that at the position $(0,0)$, where we set the axis as the original point at a depth of $10 \mathrm{~cm}$. Figure 5a shows the result of the directly simulated fluence spectrum for the given field size and the energy spectra of the incident photons. Figure $5 \mathrm{~b}$ represents the spectra convolved using the poly-energetic kernel for the given field size.

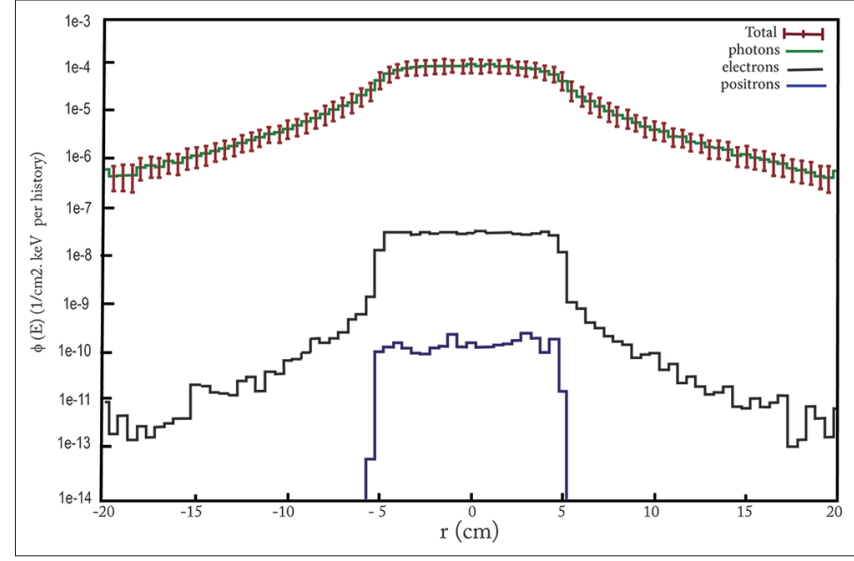

Figure 4: A profile of the fluencies of different particle categories at $10 \mathrm{~cm}$ depth underwater surface and the field size of $10 \mathrm{~cm} \times 10 \mathrm{~cm}$

One of the major drawbacks of this convolution is that the calculated kernel is based on the mono-energetic pencil kernels while the simulated spectrum is given in the form of a histogram. This explains the peaks that appear in Figure 5a because the kernel is considered as a combination of several energy peaks, which caused this difference.

Local photon spectrum is obtained by convolving PBK spectra through simulating the irradiation of monodirectional mono-energetic beam over a field of $10 \mathrm{~cm} \times 10 \mathrm{~cm}$ in an infinite water phantom. A successful database recalculation of the pencil kernels based on mono-energetic PBK is significant. The established database of the fluence spectra are divided into four categories according to their interactions: (1) uninterrupted incident photons or primary photons; (2) primary electrons that are created during primary photon interactions; (3) scattered photons which are resulted from Compton, Rayleigh, bremsstrahlung, pair production, and ionization effects interactions; and (4) scattered electrons that are created by scattered photons interactions. Although there are some singular peaks appearing in the convoluted spectrum [Figure 5a] the quantities and the form of the fluence spectra of the same volume agree well. In addition, note that the number of simulated particles in the test field of $10 \mathrm{~cm} \times 10 \mathrm{~cm}$ is still quite small. This small number of simulated particles is further evidenced by the uncertainty of the spectra, which is quite large in Figure 5a. The importance of this study is essential when dealing with more sensitive solid-state dosimeters. Those dosimeters are deviated from tissue-equivalent materials such as diodes and MOSFETs. As a result, it could be easy to tackle any induced spectral variation in these dosimeters or its covering cavities. Furthermore, it is important to analyze any local spectrum perturbation based on scattered and primary photons ${ }^{[47]}$ or scattered and primary electrons independently. So that, some modifications were employed in cavity theories considering the aforementioned photons and electrons categories and matched with our results. ${ }^{[12,47]}$ Moreover, it could be easy and fast to get correction doses factor for the sensitive dosimeters that could be linked to any local spectra perturbation. 


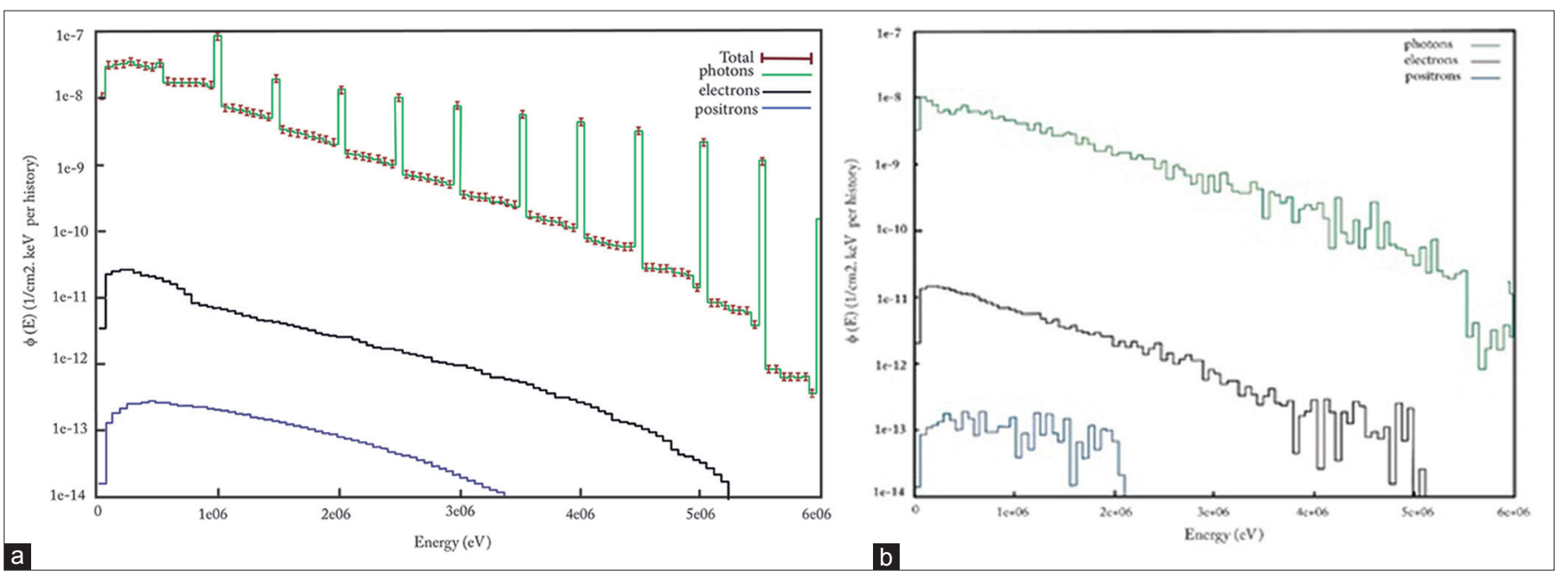

Figure 5: (a) Monte Carlo simulation results of pencil-beam fluence spectra of the voxel at the center position of the field of $10 \mathrm{~cm} \times 10 \mathrm{~cm}$ extracted from the simulation results based on convolution for each mono-energetic fluence. (b) Monte Carlo simulation results of pencil-beam fluence spectra of the voxel at the center position of the field of $10 \mathrm{~cm} \times 10 \mathrm{~cm}$ extracted out of pre-validated spectrum based on poly-energetic kernel

\section{Conclusion}

The pencil-beam approach is essential for modeling complex treatments like those of the highly modulated IMRT and volumetric modulated arc therapy fields because this approach considers the off-axis doses carefully by changing the entrance points. This study investigated establishing a PBK database based on MC algorithm to calculate convoluted spectra in homogeneous medium. Such database is classified into four categories of primary photons, primary electrons, secondary photons, and secondary electrons. The results of the comparisons between two pencil-beam fluence spectra of two different methods have been quite encouraging. In the first method, the fluence is calculated by convolution of mono-energetic fluence quantities over a field size of $10 \mathrm{~cm} \times 10 \mathrm{~cm}$. In the second method, fluence is computed by convolution of poly-energetic fluence quantities of predetermined validated spectrum over same field size. Such comparison showed a little singular peaks; however, the quantities and the form of the fluence spectra of the same volume are good fitted. Hence, it would make a considerable saving in computation time of dose calculations. This method is considered helpful for implementation of fast calculations for dose corrections into the routines of radiotherapy clinics.

Financial support and sponsorship

None.

\section{Conflicts of interest}

There are no conflicts of interest.

\section{References}

1. Tilly D, Ahnesjö A. Fast dose algorithm for generation of dose coverage probability for robustness analysis of fractionated radiotherapy. Phys Med Biol 2015;60:5439-54.

2. Carvajal MA, García-Pareja S, Guirado D, Vilches M, Anguiano M, Palma AJ, et al. Monte Carlo simulation using the PENELOPE code with an ant colony algorithm to study
MOSFET detectors. Phys Med Biol 2009;54:6263-76.

3. Ahnesjö A, van Veelen B, Tedgren ÅC. Collapsed cone dose calculations for heterogeneous tissues in brachytherapy using primary and scatter separation source data. Comput Methods Programs Biomed 2017;139:17-29.

4. Chaikh A, Kumar T, Balosso J. What should we know about photon dose calculation algorithms used for radiotherapy? Their impact on dose distribution and medical decisions based on TCP/NTCP. A review. Int J Cancer Ther Oncol 2016;4:1-12.

5. Mather ML, Collings AF, Bajenov N, Whittaker AK, Baldock C. Ultrasonic absorption in polymer gel dosimeters. Ultrasonics 2003;41:551-9.

6. Baldock C, De Deene Y, Doran S, Ibbott G, Jirasek A, Lepage M, et al. Polymer gel dosimetry. Phys Med Biol 2010;55:R1-63.

7. Vial P, Oliver L, Greer PB, Baldock C. An experimental investigation into the radiation field offset of a dynamic multileaf collimator. Phys Med Biol 2006;51:5517-38.

8. Hurley C, McLucas C, Pedrazzini G, Baldock C. High-resolution gel dosimetry of a HDR brachytherapy source using normoxic polymer gel dosimeters: Preliminary study. Nucl Instrum Methods 2006;565:801-11.

9. Knöös T. 3D dose computation algorithms. J Phys Conf Ser 2017;847:012037.

10. Reinhart AM, Fast MF, Ziegenhein P, Nill S, Oelfke U. A kernel-based dose calculation algorithm for $\mathrm{kV}$ photon beams with explicit handling of energy and material dependencies. Br J Radiol 2017;90:20160426.

11. Mainegra-Hing E, Rogers DW, Kawrakow I. Calculation of photon energy deposition kernels and electron dose point kernels in water. Med Phys 2005;32:685-99.

12. Eklund K, Ahnesjö A. Fast modelling of spectra and stopping-power ratios using differentiated fluence pencil kernels. Phys Med Biol 2008;53:4231-47.

13. Azcona JD, Barbés B, Wang L, Burguete J. Experimental pencil beam kernels derivation for $3 \mathrm{D}$ dose calculation in flattening filter free modulated fields. Phys Med Biol 2016;61:50-66.

14. Taylor PA, Kry SF, Followill DS. Pencil beam algorithms are unsuitable for proton dose calculations in lung. Int $\mathrm{J}$ Radiat Oncol Biol Phys 2017;99:750-6.

15. Eklund K, Ahnesjö A. Modeling silicon diode dose response factors for small photon fields. Phys Med Biol 2010;55:7411-23.

16. Knöös T, Wieslander E, Cozzi L, Brink C, Fogliata A, Albers D, et al. Comparison of dose calculation algorithms for treatment planning in external photon beam therapy for clinical situations. Phys Med Biol 2006;51:5785-807. 
17. Das IJ, Ding GX, Ahnesjö A. Small fields: Nonequilibrium radiation dosimetry. Med Phys 2008;35:206-15.

18. Chetty IJ, Curran B, Cygler JE, DeMarco JJ, Ezzell G, Faddegon BA, et al. Report of the AAPM Task Group No 105: Issues associated with clinical implementation of Monte Carlo-based photon and electron external beam treatment planning. Med Phys 2007;34:4818-53.

19. Partanen M, Ojala J, Niemelä J, Björkqvist M, Keyriläinen J, Kapanen M, et al. Comparison of two Monte Carlo-based codes for small-field dose calculations in external beam radiotherapy. Acta Oncol 2017;56:891-3.

20. Fonseca TC, Mendes BM, Lacerda MA, Silva LA, Paixão L, Bastos FM, et al. MCMEG: Simulations of both PDD and TPR for 6MV LINAC photon beam using different MC codes. Radiat Phys Chem 2017;140:386-91.

21. Baro J, Sempau J, Fernández-Varea JM, Salvat F. PENELOPE: An algorithm for Monte Carlo simulation of the penetration and energy loss of electrons and positrons in matter. Nucl Instrum Methods B 1995;100:31-46.

22. Sempau J, Acosta E, Baro J, Fernández-Varea JM, Salvat F. An algorithm for Monte Carlo simulation of coupled electron-photon transport. Nucl Instrum Methods B 1997;132:377-90.

23. Sempau J, Fernandez-Varea JM, Acosta E, Salvat F. Experimental benchmarks of the Monte Carlo code PENELOPE. Nucl Instrum Methods B 2003;207:107-23.

24. Salvat F, Fernández-Varea JM, Sempau J, Llovet X. Monte Carlo simulation of bremsstrahlung emission by electrons. Radiat Phys Chem 2006;75:1201-19.

25. Almansa JF, Guerrero R, Torres J, Lallena AM. Monte Carlo dosimetric characterization of the Flexisource co-60 high-dose-rate brachytherapy source using PENELOPE. Brachytherapy 2017;16:1073-80.

26. Salvat F, Fernández-Varea JM, Sempau J. PENELOPE-2008: A Code System for Monte Carlo Simulation of Electron and Photon Transport. Issy-les-Moulineaux: OECD Nuclear Energy Agency; 2008.

27. Sempau J, Badal A, Brualla L. A PENELOPE-based system for the automated Monte Carlo simulation of clinacs and voxelized geometries-application to far-from-axis fields. Med Phys 2011;38:5887-95.

28. Llovet X, Salvat F. PENEPMA: A Monte Carlo program for the simulation of X-ray emission in electron probe microanalysis. Microsc Microanal 2017;23:634-46.

29. Vella A, Munoz A, Healy MJF, Lane DW, Lockley D, Zhou JG. A fast and reliable approach to simulating the output from an X-ray tube used for developing security backscatter imaging. Proc SPIE 10388; 2017.

30. Birindelli G, Feugeas JL, Caron J, Dubroca B, Kantor G, Page J, et al. High performance modelling of the transport of energetic particles for photon radiotherapy. Phys Med 2017;42:305-12.

31. Sharma D, Sempau J, Badano A. On the efficiency of variance reduction techniques for Monte Carlo estimates of imaging noise. Med Phys 2018:45:629-34.

32. Ceberg $\mathrm{CP}$, Bjärngard $\mathrm{BE}$, Zhu TC. Experimental determination of the dose kernel in high-energy $\mathrm{x}$-ray beams. Med Phys 1996;23:505-11.

33. Mayles P, Nahum A, Rosenwald JC, edirors. Handbook of Radiotherapy Physics: Theory and Practice. Boca Raton, FL: Taylor \& Francis Group, CRC Press; 2007.

34. Storchi P, Woudstra E. Calculation of the absorbed dose distribution due to irregularly shaped photon beams using pencil beam kernels derived form basic beam data. Phys Med Biol 1996;41:637-56.

35. Ahnesjö A, Saxner M, Trepp A. A pencil beam model for photon dose calculation. Med Phys 1992;19:263-73.

36. Ulmer W, Pyyry J, Kaissl W. A 3D photon superposition/ convolution algorithm and its foundation on results of Monte Carlo calculations. Phys Med Biol 2005;50:1767-90.

37. Van Esch A, Tillikainen L, Pyykkonen J, Tenhunen M, Helminen H, Siljamäki S, et al. Testing of the analytical anisotropic algorithm for photon dose calculation. Med Phys 2006;33:4130-48.

38. Tillikainen L, Helminen H, Torsti T, Siljamäki S, Alakuijala J, Pyyry J, et al. A 3D pencil-beam-based superposition algorithm for photon dose calculation in heterogeneous media. Phys Med Biol 2008;53:3821-39.

39. Knöös T, Ceberg C, Weber L, Nilsson P. The dosimetric verification of a pencil beam based treatment planning system. Phys Med Biol 1994;39:1609-28.

40. Knöös T, Ahnesjö A, Nilsson P, Weber L. Limitations of a pencil beam approach to photon dose calculations in lung tissue. Phys Med Biol 1995;40:1411-20.

41. Hurkmans C, Knöös T, Nilsson P, Svahn-Tapper G, Danielsson H. Limitations of a pencil beam approach to photon dose calculations in the head and neck region. Radiother Oncol 1995;37:74-80.

42. Weber L, Ahnesjö A, Nilsson P, Saxner M, Knöös T. Verification and implementation of dynamic wedge calculations in a treatment planning system based on a dose-to-energy-fluence formalism. Med Phys 1996;23:307-16.

43. Hurkmans C, Knöös T, Nilsson P. Dosimetric verification of open asymmetric photon fields calculated with a treatment planning system based on dose-to-energy-fluence concepts. Phys Med Biol 1996;41:1277-90.

44. Hansson H, Björk P, Knöös T, Nilsson P. Verification of a pencil beam based treatment planning system: Output factors for open photon beams shaped with MLC or blocks. Phys Med Biol 1999;44:N201-7.

45. Sheikh-Bagheri D, Rogers DW. Sensitivity of megavoltage photon beam Monte Carlo simulations to electron beam and other parameters. Med Phys 2002;29:379-90.

46. Almond PR, Andreo P, Mattsson O, Nahum AE, Roos M. The Use of Plane-Parallel Ionization Chambers in High-Energy Electron and Photon Beams. An International Code of Practice for dosimetry. Technical Reports Series No. 381. Vienna: IAEA: IAEA; 1997.

47. Yin Z, Hugtenburg RP, Beddoe AH. Response corrections for solid-state detectors in megavoltage photon dosimetry. Phys Med Biol 2004;49:3691-702.

\section{BIOGRAPHIES}

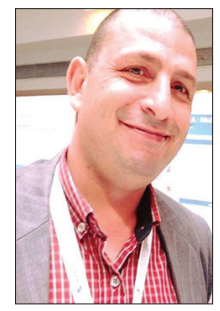

Hossam Donya Dr. Hossam Donya works as assistant professor at the Department of Physics, Faculty of Science, King Abdulaziz University, Jeddah, Saudi Arabia. His permanent affiliation is also at the Department of Physics, Faculty of Science, Menoufia University, Shibin El-Koom, Egypt

Email: hdunia@kau.edu.sa 